\title{
Article \\ Optimal Scheduling of Microgrid Considering the Interruptible Load Shifting Based on Improved Biogeography-Based Optimization Algorithm
}

\author{
Bo Li, Hongsheng Deng * (D) and Jue Wang \\ College of Information Science and Engineering, Dalian Polytechnic University, Dalian 116034, China; \\ libolb@dlpu.edu.cn (B.L.); wangjue@dlpu.edu.cn (J.W.) \\ * Correspondence: dhongsheng1998@gmail.com
}

check for

updates

Citation: Li, B.; Deng, H.; Wang, J. Optimal Scheduling of Microgrid Considering the Interruptible Load Shifting Based on Improved

Biogeography-Based Optimization Algorithm. Symmetry 2021, 13, 1707 https: / / doi.org/10.3390/ sym13091707

Academic Editors: Raúl

Baños Navarro and Alfredo Alcayde

Received: 26 August 2021

Accepted: 14 September 2021

Published: 15 September 2021

Publisher's Note: MDPI stays neutral with regard to jurisdictional claims in published maps and institutional affiliations.

Copyright: (c) 2021 by the authors. Licensee MDPI, Basel, Switzerland. This article is an open access article distributed under the terms and conditions of the Creative Commons Attribution (CC BY) license (https:// creativecommons.org/licenses/by/ $4.0 /)$.

\begin{abstract}
A microgrid is an efficient method of uniting distributed generations. To ensure the applicability and symmetry of the microgrid, the environmental benefits and economic costs are considered to comprehensively model the optimal operation of the microgrid under the grid-connected operation mode, at the same time, considering the effect of interruptible load on the operating cost of the microgrid, the power shifting for interruptible load is attempted on the basis of battery storage capacity. By adaptively adjusting the migration rate using the habitat suitability index of a normalized individual and adding a certain differential perturbation to the migration operator of the migration mechanism, an improved biogeography-based optimization algorithm is proposed and the microgrid optimization dispatching algorithm based on the improved biogeography-based optimization is applied. The advancement and effectiveness of the proposed algorithm and model is verified by the example, and the simulation results indicate that the implementation of the power dispatching scheme proposed in this paper can effectively reduce the total cost of the system. Moreover, the proper consideration of shifting interruptible load, the effective load management and guiding the electricity consumption behavior of users are of certain significance for optimizing the utilization of renewable energy and improving the system efficiency and economy.
\end{abstract}

Keywords: microgrid; optimal scheduling; biogeography-based optimization algorithm; adaptive determination mechanism of migration rate; dynamic migration mechanism; power shifting; interruptible load

\section{Introduction}

With global warming and the depletion of energy, renewable clean energy, which includes wind and solar power, has gradually attracted people's attention. However, uncontrollable factors of renewable energy caused by the randomness and intermittence of wind energy and light intensity seriously affect the stability and reliability of the grid operation. At the same time, it is difficult for a power system with only several renewable energy generations to meet the load demand in isolated islands. Hence, a microgrid, which combines distributed micro power supply, load unit, energy storage unit and control equipment, can deal with the above problems well. It has become a hot research topic for many scholars to reasonably optimize a microgrid system to achieve economic and environmental optimization.

Firstly, in the field of microgrid energy system optimization, research topics in large numbers focus on the optimization of the output power or economic model [1-3], the improvement of evolution strategy or the optimization of the control system [4]. To be specific, distributed power generation scheduling problems are usually nonlinear and non-convex multi-objective optimization problems [5]. It is difficult for traditional optimization algorithms and mathematical methods dealing with convex functions to solve 
such problems. Most intelligent optimization algorithms, which include the particle swarming algorithm (PSO) [6-8], the genetic algorithm (GA) $[9,10]$ and the bacterial foraging algorithm (BFA) [11], have different optimization effects in different fields. In recent years, intelligent optimization algorithms in large numbers have been widely used in the power dispatching of a microgrid. With the continuous efforts of many scholars, optimization algorithms have developed different evolution strategies. In [12], the optimization model of a microgrid, considering the economy, environment and system operation risks, is built, and a multi-objective particle swarm algorithm based on niche technology is used to deal with this model. In [13], a microgrid system combined heat and power is presented, which reduces the operating cost of the microgrid by recycling the waste heat in the system. In [14], to better deal with the energy efficiency and pollutant emission problems of the microgrid, fuzzy processing technology and tabu search are introduced and an improved particle swarm optimization algorithm is proposed. In [15], the traditional niche technology is improved in the elimination rule of individual fitness, in order to propose an improved genetic optimization algorithm. In [16], the environmental treatment cost, maintenance cost and operating cost of the microgrid system are considered separately, and the multi-objective optimization model is converted into a single-objective optimization model by adding the weight coefficient, and the optimization results obtained by different weight coefficients are analyzed. In [17], the optimal configuration model is established, which included the investment cost of equipment, environmental protection cost, operation cost, maintenance cost and fuel cost, and the improved bacterial foraging algorithm (BFA) is used to deal with the problem of optimal configuration for the hybrid island operation of a microgrid. In [18], considering several objectives including economic benefits, network loss and environmental costs, by introducing elite strategy, reverse learning mechanism and global memory, a microgrid optimization operation method based on advanced gravitational search algorithm is applied. In [19], considering the security, economy and environmental protection of the microgrid, the three indicators, which include the microgrid system's voltage stability, active power loss and gas pollution, are quantitatively evaluated, and the microgrid optimization model is established; the strength Pareto evolutionary algorithm proposed solves this model. In [20], based on the theory of chance-constrained programming, a dynamic economic dispatch model of the microgrid with wind farms considering microgrid operation risk constraints is established, and a hybrid intelligent optimization algorithm combining sequence operation theory and a genetic algorithm is proposed to solve the model. In [21], by modeling the uncertainty of light power and wind power, an Adaptive Modified Firefly Algorithm (AMFA) is proposed. Many of the various algorithms mentioned above consider economic and environmental factors, but they do not consider load optimization strategies. For this reason, a multi-objective optimization strategy considering interruptible load shifting is proposed in this paper.

A biogeography-based optimization (BBO) algorithm, proposed by American scholar Dan Simon [22], is a swarm intelligence optimization algorithm based on biogeography theory. Compared with the PSO and GA algorithms, BBO has some advantages in convergence performance, and the ability of this algorithm in optimization has currently been verified by expert scholars through experimental studies. However, the basic BBO still suffers from premature convergence and faces the problem of local optimization; it still needs to be improved to further enhance its optimization performance. The optimization of the typical daily microgrid system in summer involves the output power of a micro gas turbine, photovoltaic array, fuel cell, storage battery and wind turbine. An optimal scheduling model of the microgrid considering the economic and environmental protection under the grid-connected operation mode is established on the premise of meeting the load demand and system constraints. Meanwhile, for the consumer side, considering the symmetry of interruptible load and the operating cost in the microgrid system, the power shifting for interruptible load is attempted on the basis of the battery storage capacity in this paper. Through simulation experiments, the comprehensive optimal scheduling of the microgrid is obtained, and compared with the basic $\mathrm{BBO}$ and other two algorithms, 
the performance of the proposed method surpasses the other methods mentioned above, which provides an effective means to solve the optimal operation of the microgrid with an intelligent optimization algorithm. The proper consideration of shifting interruptible load, the effective load management and guiding the electricity consumption behavior of users are of certain significance for optimizing the utilization of renewable energy and improving system efficiency and economy.

\section{Multi-Objective Optimization Operation of Microgrid under Grid-Connected Mode}

The microgrid power generation units used in this paper are mainly divided into renewable energy units and non-renewable energy units. Among them, renewable energy power units include photovoltaic cells (PV) and wind turbines (WT) with uncontrollable power generation; non-renewable energy units are composed of micro gas turbines $(M T)$ and fuel cells $(F C)$ with controllable power generation. Through the coordinated use of the above units under different constraints, the optimization of environmental benefit and economic cost can be obtained.

\subsection{System Power Generation Models}

\subsubsection{Model of WT Generator}

The specific output formula of a wind turbine is shown in Equation (1):

$$
P_{W T}=\left\{\begin{array}{l}
0, v \leq v_{c i}, v>v_{c o} \\
P_{v}, v_{c i}<v \leq v_{c o}
\end{array}\right.
$$

where $v$ is the current wind speed and $v_{c i}$ and $v_{c o}$ are the minimum and maximum wind speeds required for the normal operation of the wind turbine. When the current wind speed is between $v_{c i}$ and $v_{c o}, P_{v}$ is the output of the WT.

\subsubsection{Model of PV Generator}

The output of photovoltaic generator, which is based on light intensity and ambient temperature, is shown in Equation (2) as follows:

$$
P_{P V}=P_{S} \frac{S_{A}}{S_{0}}\left(1+k\left(T_{c}-T_{r}\right)\right)
$$

In Equation (2), $P_{S}$ represents the maximum output power of $P V ; S_{A}$ and $S_{o}$ represent the ambient light intensity during the scheduling period and the light intensity under the rated power, respectively; $k$ represents the ambient temperature coefficient; $T_{C}$ represents the actual temperature of the $P V$ board during the dispatch period; and $T_{r}$ represents the reference temperature under standard conditions.

\subsubsection{Model of MT Fuel Cost}

The micro gas turbine (MT) fuel cost can be calculated as Equation (3) as follows:

$$
F_{M T}=C_{f u e l} \frac{1}{L} \frac{P_{M T}}{\eta_{M T}}
$$

where $F_{M T}$ is the $M T$ fuel cost; $C_{f u e l}$ and $L$ are the real-time price of fuel gas and the low calorific value of fuel gas, respectively; and $P_{M T}$ and $\eta_{M T}$ are the actual output and the working efficiency of the micro gas turbine, respectively.

\subsubsection{Model of Fuel Cell Cost}

A fuel cell is a piece of electrochemical equipment that reacts hydrogen with oxygen or other oxides. The principle of fuel cell power generation is that the chemical energy of fuel is converted into electrical energy [23]. The main discharge of a fuel cell is water, 
there are no pollutants such as $\mathrm{CO}$ and $\mathrm{NO}_{\mathrm{x}}$, and its power generation efficiency is high; therefore, it is widely used in the field of power systems.

The fuel cost model of the fuel cell is similar to the fuel gas cost model of the micro gas turbine in Equation (3); therefore, it can be given by the following:

$$
F_{F C}=C_{f u e l} \frac{1}{L} \frac{P_{F C}}{\eta_{F C}}
$$

where $F_{F C}$ represents the fuel cell cost, and $P_{F C}$ and $\eta_{F C}$ represent the output and working efficiency of the fuel cell, respectively.

\subsubsection{Model of Storage Battery}

Considering the uncertainty of renewable energy in the optimal scheduling of the microgrid, such as a $W T$ generator and a $P V$ generator, storage batteries are added to maintain the stability of the microgrid operation and minimize the economic loss by overload. The model of the state of charge of a battery $(S O C)$ is established, which represents the radio of the current remaining power of the battery to the total capacity of the battery. The specific formula is expressed as follows.

The charging formula of storage battery can be calculated as follows:

$$
S O C_{t+1}=(1-\sigma) S O C_{t}+\left(P_{E S, t} \Delta t \eta_{I N}\right) / C
$$

where $S O C_{t}$ and $S O C_{t+1}$ are the state of charge of the battery at time $t$ and time $t+1 ; \sigma$ represents the self-discharge coefficient of the storage battery; $P_{E S, t}$ is the charge-discharge power at time $t$. When the battery is charging, $P_{E S, t}>0$, when the battery stops, $P_{E S, t}=0$, otherwise $P_{E S, t}<0 . \eta_{I N}$ represents the charge efficiency of the storage battery and $C$ is the maximum charging capacity of the storage battery within unit time $\Delta t$.

The discharging formula of storage battery can be calculated as follows:

$$
S O C_{t+1}=(1-\sigma) S O C_{t}-P_{E S, t} \Delta t /\left(C \eta_{\text {OUT }}\right)
$$

where all the symbols represent the same as they did in Equation (5) and $\eta_{O U T}$ represents the discharge efficiency of the storage battery.

\subsection{The Key Technologies for Optimal Dispatch of Microgrid}

The aim of the optimal dispatch of the microgrid is to achieve the minimum economic cost and environmental cost by rationally dispatching the output power of different distributed power generation units and satisfying various power constrains and load requirements.

\subsubsection{The Objective Function}

- Cost of the operating

As one of the most important optimization indexes in the microgrid optimization dispatching, the microgrid operating cost plays an important role in measuring the feasibility and applicability of the microgrid system. It includes the fuel cost and the maintenance cost of each generator, as well as the online electricity cost. The specific formula can be defined as the following Equation (7):

$$
\min F_{1}=\sum_{t=1}^{T} \sum_{i=1}^{N}\left(C_{F, i, t}+C_{O M, i, t}+n C_{G, t}\right)
$$

where $F_{1}$ represents the total cost of system operation; $T$ is the number of unit scheduling intervals of the system during a scheduling period, in this paper, $T$ is $24 ; N$ is the total number of various power generators; $C_{F, j, t}$ and $C_{O M, j, t}$ are the fuel cost and the maintenance cost of the $i$-th generator in the $t$-th time interval, respectively; $C_{O M, I, t}=k_{O M, I} P_{i, t}, k_{O M, I}$ 
and $P_{i, t}$ are the loss coefficient and the output of the $i$-th generator in the $t$-th time interval, respectively; and $C_{G, t}$ represents the online electricity cost at time $t$. When the microgrid is running in the grid-connected operation mode, $n=1$.

- Cost of pollution

With global warming, people have comprehensively promoted the sustainable development strategy, and the cost of pollution has become an important part of the total cost of the microgrid. The pollution index in this paper is the pollution degree judgment made by the pollution of $\mathrm{CO}, \mathrm{SO}_{2}$, Nox and other pollutants discharged by non-clean energy units, such as micro turbines and fuel cells. Since clean energy units, which include photovoltaic cells $(P V)$ and wind power $(W T)$, do not discharge pollution gas, they can be neglected in this pollution cost. The minimum pollution cost formula can be given by the following:

$$
\min F_{2}=\sum_{t=1}^{T} \sum_{j=1}^{M} C_{j} \sum_{i=1}^{N} K_{i j} P_{i, t}
$$

where $F_{2}$ represents the total cost required to treat the pollutants emitted by the microgrid; $M$ represents the number of pollutant types; $C_{j}$ represents the treatment cost corresponding to the pollutant $j ; K_{i j}$ represents the treatment coefficient of the pollutant $j$ discharged by the $i$-th generator; $P_{i, t}$ represents the output of the $i$-th generator during the $t$-th time period.

In addition, the calculation formula of the comprehensive cost can be obtained by linearly weighting the operating cost and the environmental governance cost, which transforms the multi-objective non-convex optimization problem into a single-objective optimization problem for the microgrid.

\subsubsection{Constraints}

The above models should satisfy the following constraints:

- The total power constraint;

$$
\sum_{i=1}^{N} P_{i, t}+P_{E S, t}+P_{G r i d, t}-P_{L, t}=0
$$

where $P_{i, t}$ represents the output power of the $i$-th micro energy unit at time $t ; P_{E S, t}$ represents the charge-discharging power of the storage battery at time $t$, when the battery is discharged, $P_{E S, t}<0 ; P_{\text {Grid,t }}$ represents the interactive power between the large grid and the microgrid at time $t$. When the load is overloaded, the microgrid purchases electric energy from the large grid, $P_{G r i d, t}>0 ; P_{L, t}$ represents the total power consumption on the load side at time $t$.

- The output power constraint of micro energy generator unit;

$$
P_{i \min } \leq P_{i} \leq P_{i \max }
$$

where $P_{i \min }$ and $P_{i \max }$ represent the upper limit and the lower limit of the $i$-th unit output.

- The transmission power constraint;

$$
0 \leq P_{\text {Grid }} \leq P_{\text {Gridmax }}
$$

where $P_{\text {Gridmax }}$ is the maximum allowable transmission power between the microgrid and the distribution grid.

- The climbing rate limit;

The constrain of load increases is as follows:

$$
P_{i, t}-P_{i, t-1} \leq R_{\text {iup }}
$$


The constrain of load reduction is as follows:

$$
P_{i, t-1}-P_{i, t} \leq R_{\text {idown }}
$$

As can be seen from Equations (12) and (13), the climbing speed of the $i$-th generator is bounded by the upper $R_{\text {iup }}$ and lower $R_{\text {idown }}$ generation limits.

- $\quad$ Energy storage battery constrains.

Considering the security of the storage battery, its state of charge must be bounded within the minimum and maximum.

$$
S O C_{i \min } \leq S O C_{i, t} \leq S O C_{i \max }
$$

where $S O C_{i \min }$ and $S O C_{i \max }$ are the allowable minimum and maximum battery states of the charge.

\subsubsection{The Objective Function Added the Interruptible Load Shifting}

The operation optimization of the microgrid is the basis for ensuring the high-quality and efficient operation of the microgrid. In the above, the comprehensive cost of microgrid optimal operation is determined by the combination of operating costs and environmental costs. Then, for the consumer side, considering the effect of interruptible load on the operating cost of the microgrid, the power shifting for interruptible load is attempted with certain constraints.

According to the actual situation, the load can be divided into non-interruptible load and interruptible load. The microgrid needs to meet uninterruptible load, and the interruptible load can attempt to shift to the time when the original demand for electricity is lower. Since the shifting of interruptible load can have certain impact on the use of electricity for the original user side, the amount of load involved in the shifting and the time span of shifting are both issues to be considered. Consequently, considering the impact on the user side in terms of the total amount of power shifting and the shifting time, a comprehensive evaluation objective function with the power shifting of the interruptible load is established in this paper, as shown in the following Equation (15):

$$
F=\left(F_{1}+F_{2}\right)\left(1+\frac{P_{L S}}{P_{L I}+P_{L U}}\right)(1+\alpha T s)
$$

where $F$ is the comprehensive evaluation objective function with interruptible load shifting. $P_{L S} /\left(P_{L I}+P_{L U}\right)$ is the penalty term of the total shifting load on the total optimization cost. Only when the optimization cost after load shifting is better than the original cost after penalty, can power shifting be carried out. $P_{L S}$ is the interruptible load involved in shifting, $P_{L I}$ is the maximum shifting load of daily interruptible load, $P_{L U}$ is the demand power of non-interruptible load and $P_{L I}+P_{L U}=P_{L}$. Obviously, when the load is not shiftable, the comprehensive evaluation objective function $F=F_{1}+F_{2}$, which is equal to the comprehensive optimization cost mentioned above. $\alpha$ is the adjustment factor of shifting time, and Ts is the total shifting time; the pseudo code for the total shifting time is shown in Algorithm 1. 
Algorithm 1 The Calculation of the Total Shifting Time.

1. Let $P_{L S I_{i}}$ be the $i$-th load shifted in and $P_{L S O_{j}}$ be the $j$-th load shifted out, where $i$ $\in\left[1, i_{\max }\right], j \in\left[1, j_{\max }\right], I_{i}$, and $O_{j}$ are the current time corresponding to the respective load, $I_{i}, O_{j} \in(0,24)$.

2. Then, $\sum_{i=1}^{i_{\max }} P_{L S I_{i}}=\left|\sum_{j=1}^{j_{\max }} P_{L S O_{j}}\right|=P_{L S} \leq P_{L I} ;$ set $P_{L S m}$ as the maximum load of single time shifting; the formulas $P_{L S I_{i}} \leq P_{L S m}$ and $\left|P_{L S O_{j}}\right| \leq P_{L S m}$ need to be satisfied.

3. Set $i=1, j=1$.

4. Calculate the cumulative sum of $P_{\mathrm{LSI}_{i}}$ and add to $P_{\mathrm{LSO}_{j}}$ to satisfy

$\sum_{i=1}^{i_{\text {max }}} P_{\mathrm{LSI}_{i}}+P_{\mathrm{LSO}_{j}} \geq 0$; record $i_{m_{j}}$.

5. Then, the shifting time $t_{j}$ of the load shifted corresponding to the $j$-th load shifted in is $t s_{j}=O_{j}-\frac{1}{i_{m_{j}}-i+1} \sum_{i}^{i_{m_{j}}} I_{i}$.

6.

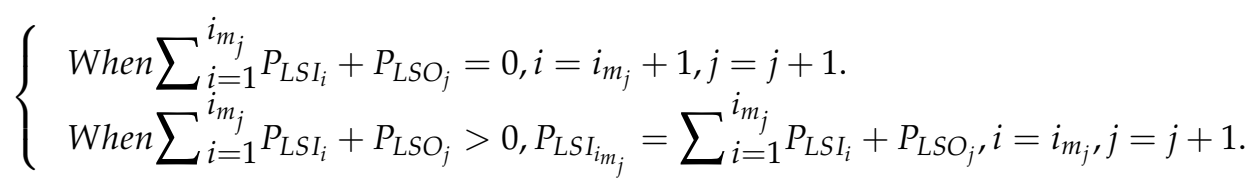

7. Repeat steps $4-6$, until $t s_{j_{\max }}$ is obtained.

Then, the total shifting time $T s=\sum_{j=1}^{j_{\max }} t s_{j}$.

Considering the degree of effect of the power shifting time on the user side, the calculation of the total shifting time Ts is added. According to the formula, compared with the original power demand time and the power consumption time after shifting, the more time that is shifted, the greater the total shifting time Ts is, and vice versa. For the time factor $\alpha$, the greater the time factor $\alpha$ is, the higher the penalty degree of shifting time Ts on the comprehensive operating cost is, which can force the power to shift to the closer time of the original power demand time. However, when the value of $\alpha$ reaches a certain threshold, even though the comprehensive operating cost of the microgrid after power shifting can be lower, the microgrid scheduling scheme with power shifting cannot be obtained due to the penalty relation of shifting time Ts. Therefore, the objective function, which comprehensively considers the operating cost, environmental cost and user-side demand of the microgrid, is established on the basis of the mutual coordination of the various distributed generation and full consideration of battery charging and discharging capacity, which can more effectively maximize the use of renewable clean energy and, thus, realize the maximum operation benefit of the microgrid.

\section{Biogeography-Based Optimization Algorithm}

Biogeography is an independent discipline that studies the temporal and spatial distribution of biological communities and their components, which originated in the 19th century. Scholars in large numbers around the world have made in-depth research on it, and numerous mathematical models for the distribution, migration and extinction of biological communities have been proposed. Inspired by these mathematical models, biogeography-based optimization (BBO) was proposed by American academic Dan Simon in 2008. According to the mathematical model of species migration, $\mathrm{BBO}$ depends on the simulation of a species migration mechanism among habitats to achieve the goal of finding the global optimal solution. 


\subsection{The Basic Biogeography-Based Optimization Algorithm}

Nowadays, the BBO algorithm is involved in various fields, including biology [24], environmental science [25], etc. In BBO, the habitats of species in biogeography correspond to the possible individuals of optimization problems, the suitability index variables (SIV) in the model correspond to individual variables, and the habitat suitability index (I) corresponds to the fitness function of evaluating the quality of a solution set. When solving optimization problems, multiple habitats are randomly generated as the initial solution of the optimization problem. Through the information exchange of species migration between habitats, the species diversity of habitats is improved, the I of habitats is improved and then the optimal solution of the problem is obtained. The key steps of BBO are as follows: initialization, migration and mutation.

- Initialization

The parameters of the BBO algorithm are initialized, including the maximum number of species per habitat $S_{\max }$, the maximum allowable immigration rate $I$ and emigration rate $E$ and the maximum mutation rate $m_{\max }$. The $\mathrm{BBO}$ algorithm randomly generates $N$ habitats as the initial population using Equation (16), and each habitat as a potential solution contains $D$-dimensional solution variables. Afterwards, the I for each habitat is calculated.

$$
x_{i j}=x_{j, \min }+\operatorname{rand}\left(x_{j, \max }-x_{j, \min }\right)
$$

where $x_{i j}$ is the $j$-th dimensional solution variable of habitat $x_{i}, i \in[1,2, \ldots, N P], j \in[1,2$, $\ldots, D]$; and $x_{j, \max }$ and $x_{j, \min }$ are the lower and upper limits of the $j$-th dimensional variable, respectively.

- Migration

The $\mathrm{BBO}$ algorithm exchanges information with other habitats through the migration mechanism, and then the solution space is searched in a wide area. Based on the basic principles of biogeography, the I of habitat is directly proportional to species diversity, and the habitat with a higher I can accommodate more species. Therefore, there is the mapping relationship between habitat $x_{i}$ and the number of species $S_{i}$. After reordering $x_{i}$ according to its corresponding I from high to low, the original habitat $x_{i}$ is given a new $i$. The number of species $S_{i}$ of $x_{i}$ after ranking is calculated using Equation (17).

$$
S_{i}=S_{\max }-i
$$

As can be seen from the mechanism of biogeographical species migration, the specific formula of the immigration rate $\lambda_{i}$ and the emigration rate $\mu_{i}$ of habitat $x_{i}$ can be given by the following:

$$
\left\{\begin{array}{l}
\lambda_{i}=I\left(1-\frac{S_{i}}{S_{\max }}\right) \\
\mu_{i}=\frac{E \cdot S_{i}}{S_{\max }}
\end{array}\right.
$$

In the migration operation, firstly, the habitat $x_{i}$ to be migrated is determined according to the migration rate $\lambda_{i}$. A random number between $(0,1)$ is generated, if it is less than $\lambda_{i}$, then the habitat $x_{i}$ is that to be moved in. Next, for each dimension of $x_{i}$, the habitat with which to migrate needs to be determined. Among the remaining $N-1$ habitats, $x_{k}$ is determined by roulette according to their migration rate $\mu_{k}$. Finally, the solution variables of habitat $x_{i}$ are replaced by the solution variables of the selected habitat $x_{k}$. 


\section{- Mutation}

A habitat may mutate its I due to a catastrophic event and the BBO algorithm uses a mutation operation to simulate the rapid change of I in a habitat caused by such an emergency. According to the immigration rate $\lambda_{i}$ and the emigration rate $\mu_{i}$, the probability $P_{i}$ of species number $S$ in habitat $x_{i}$ is calculated using Equation (19).

$$
P_{i}= \begin{cases}-\left(\lambda_{i}+\mu_{i}\right) P_{i}+\mu_{i+1} P_{i+1}, & S_{i}=0 \\ -\left(\lambda_{i}+\mu_{i}\right) P_{i}+\lambda_{i-1} P_{i-1}+\mu_{i+1} P_{i+1}, & 1 \leq S_{i} \leq S_{\max }-1 \\ -\left(\lambda_{i}+\mu_{i}\right) P_{i}+\lambda_{i-1} P_{i-1}, & S_{i}=S_{\max }\end{cases}
$$

The mutation rate $m_{i}$ of habitat $x_{i}$ is calculated using Equation (20).

$$
m_{i}=m_{\max }\left(1-\frac{P_{i}}{P_{\max }}\right)
$$

where $m_{\max }$ is the maximum mutation rate of the habitat and $P_{\max }$ is the maximum probability of all species.

\subsection{Improved BBO Algorithm}

- Adaptive determination mechanism of migration rate

The determination of the immigration and emigration rate in $\mathrm{BBO}$ can only reflect the order of individual quality, while ignoring the evolutionary state of the current population and failing to rationally assess the quality of individuals. Once individuals have large quality gaps or are unevenly distributed, it is easy to make an incorrect assessment of the degree of the individual's quality, resulting in the beneficial information of the better individual unable to be retained, or the poor individual who participated early in evolution. Based on the above question, this paper uses the I value of normalized individuals to adjust the immigration rate adaptively, as shown in Equation (21).

$$
\left\{\begin{array}{l}
\lambda_{i}=\left(\frac{F_{i}-F_{\min }}{F_{\max }-F_{\min }}\right) \\
\mu_{i}=1-\lambda_{i}
\end{array}\right.
$$

where $F_{i}$ is the I of habitat $x_{i}$ and $F_{\max }$ and $F_{\min }$ are the maximum and minimum value of $I$ in the current population. This method can solve the problem that individual quality cannot be monitored in real time while ensuring the effective use of information about individuals in the current population and evaluate the individuals to be immigrated or emigrated more accurately.

\section{- Dynamic migration mechanism}

The migration strategy of BBO as an important part of its evolution mechanism, to a large extent, affects the performance of the algorithm. However, its migration method of directly replacing the SIV of the original solution with the SIV to be emigrated, may lead to a certain blindness in migration, and the poor ability of mining new solutions, which can easily cause premature convergence. Therefore, a new dynamic migration mechanism is employed to solve this problem, which uses an adaptive migration rate designed in the previous section to adjust the degree of perturbation of the differential vector for mining new solutions, in order to achieve adaptive perturbation around the SIV to be emigrated at different evolutionary periods, thus enhancing the search capability of the migrating algorithm, as shown in Equation (22).

$$
x_{i j}=x_{k j}+\left(r_{\min }+\lambda_{i} \times\left(r_{\max }-r_{\min }\right)\right) \times\left(x_{a j}-x_{b j}\right)
$$

where $x_{k}$ is the habitat to be emigrated; $a$ and $b$ are random numbers among $[1, N P]$; then, $x_{a j}$ and $x_{b j}$, respectively, represent the $j$-th dimensional variables of the $a$-th and the $b$-th habitat; and $r_{\max }$ and $r_{\min }$ are the maximum and minimum disturbance degree, respectively. 


\subsection{The Algorithm Flow}

The specific operation process of microgrid optimization based on the improved $\mathrm{BBO}$ (IBBO) algorithm can be clearly expressed through the following eight steps.

Step 1. Describe the actual power data and system parameters of the microgrid, such as the output of photovoltaic and wind turbine, power demand on the side, etc. In addition, the output of each distributed power generation and battery needs to be limited. Then, define the general parameters of the IBBO algorithm, including $S_{\max }, I, E$ and $m_{\max }$.

Step 2. Set the population size $N P$, and random population $H=\left\{x_{i}, I=1,2, \ldots, N P\right\}$. Each individual in the population $H$ is determined by the micro power supplies in different scheduling times and the dimensions of the individual are equal to $(N+1) \times t$. $N$ represents the total number of those distributed generators, and $t$ represents the total time for the optimal dispatch of the microgrid. The last row is the shifting amount of the corresponding scheduling time when considering load shifting. Certainly, when the load is not shiftable, no matter what the row value is, it does not affect the output results of each micro power supply at each scheduling time.

Step 3. Evaluate the HSI of the current habitat individuals, eliminate individuals that do not meet the constraints and sort the remaining habitat individuals in ascending order according to HSI and use them as population $H$.

Step 4. From Equation (21), calculate the value of $\lambda_{i}$ and $\mu_{i}$ for each habitat $x_{i}$ in the population $H$ separately.

Step 5. Substitute the parameters $\lambda_{i}$ and $\mu_{i}$ into Equation (22) to obtain the migration.

Step 6. Determine the mutation rate $m_{i}$ and species probability $P_{i}$ in each habitat. According to the mutation rate of each habitat, make mutation for species.

Step 7. Judge whether the obtained population meets the requirements, if not, continue to the next iteration and go back to stage three, else execute the next stage.

Step 8. Print the optimal habitat population $H$.

\section{Simulation Results and Discussion}

\subsection{Calculation Parameters and Power Data Settings}

By analyzing and discussing the operation of the microgrid under grid-connected mode to verify the authenticity and effectiveness of the model established above.

The improved biogeography-based optimization algorithm mentioned in this paper, which involves several units such as wind turbines, photovoltaic cells, micro gas turbines, batteries and interaction power with the main grid, is applied to the optimization of multiple objectives in the microgrid with hours as the time unit, the specific parameters are shown in Table 1. Other parameters of the microgrid system include the maximum allowable charge capacity of the storage battery, which is $250 \mathrm{kWh}$; the maximum interaction power, which is $50 \mathrm{kw}$; to be specific, microgrid purchases electricity from the large grid as positive and sells it as negative. By investigating the local climate and electricity consumption and selecting a common summer weather, we learn that the peak power consumption of the day is at 12:00 and 20:00, and the maximum can reach $160 \mathrm{~kW}$. In addition, other forecast data, such as light intensity, temperature and wind speed are given in Figure 1. It can be analyzed from Figure 1 that from 12:00 to 13:00 that day, the light intensity reached the maximum value of $1000 \mathrm{~W} / \mathrm{m}^{2}$, the temperature also reached the maximum value of $31^{\circ} \mathrm{C}$, and the wind speed reached the maximum value of $9.2 \mathrm{~m} / \mathrm{s}$ at 10:00. To ensure the maximum utilization of renewable energy and maximum economic and environmental benefits, wind turbines and photovoltaic cells will continue to run under the maximum power point trace mode (MPPT); the output curves are given in Figure 2. In one day, the output power of the wind turbine is the maximum at 10:00, and the photovoltaic power generation is the maximum at 12:00, and the values are 46 and $36 \mathrm{~kW}$, respectively. From [12], we can know the real-time electricity price and pollution penalty standards and other electricity data. There are the following specific values of parameters in $\mathrm{BBO}$ and IBBO algorithms: the initial value of the population size NP is set to 100 , and the maximum number of species $S_{\max }$ is also 100, upper limit of immigration 
rate and emigration rate $I=E=1.0$, upper limit of mutation rate $m_{\max }=0.005$ and the number of iterations is limited to 500. Compared with the IBBO algorithm, the population size of the other algorithms is also set as 100 , and the other parameter settings can be found in $[10,11,22]$.

Table 1. Parameters of each Generators.

\begin{tabular}{ccccc}
\hline DG & \multicolumn{2}{c}{ Output Power/kW } & Depreciable Life/a & $\begin{array}{c}\text { Operation and Maintenance } \\
\text { Coefficient (USD/kW.h) }\end{array}$ \\
\cline { 2 - 3 } & $\boldsymbol{P}_{\max }$ & $\boldsymbol{P}_{\min }$ & & 0.0042 \\
$W T$ & 50 & 0 & 20 & 0.0014 \\
$P V$ & 40 & 0 & 10 & 0.0064 \\
BAT & 30 & -30 & 10 & 0.0042 \\
$F C$ & 40 & 8 & 10 & 0.0059 \\
MT & 65 & 14 & & \\
\hline
\end{tabular}
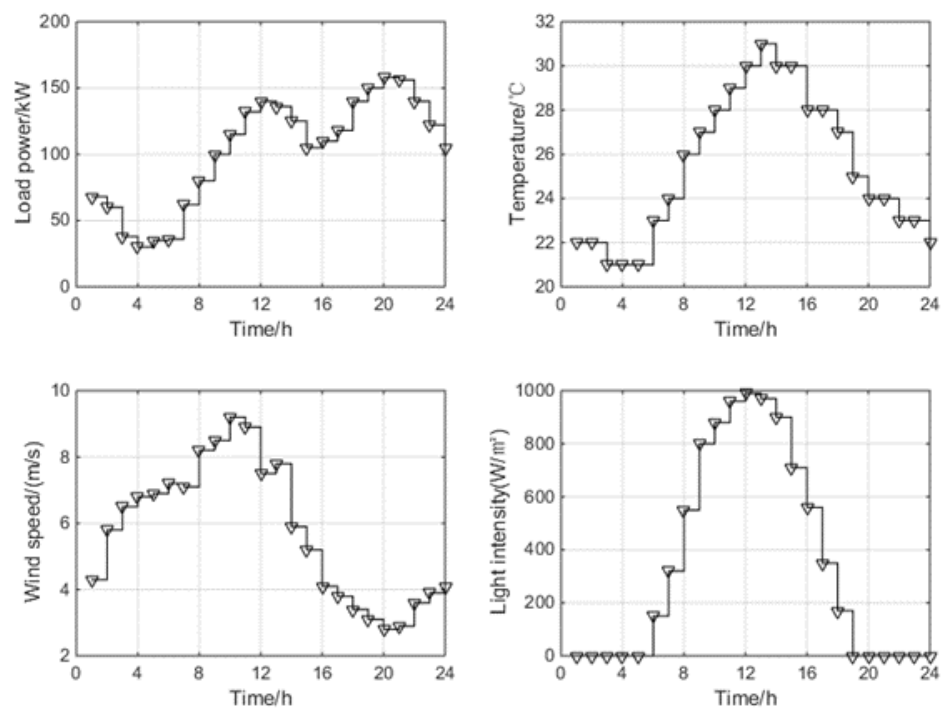

Figure 1. Predicted value of load power, wind speed, temperature and light intensity.
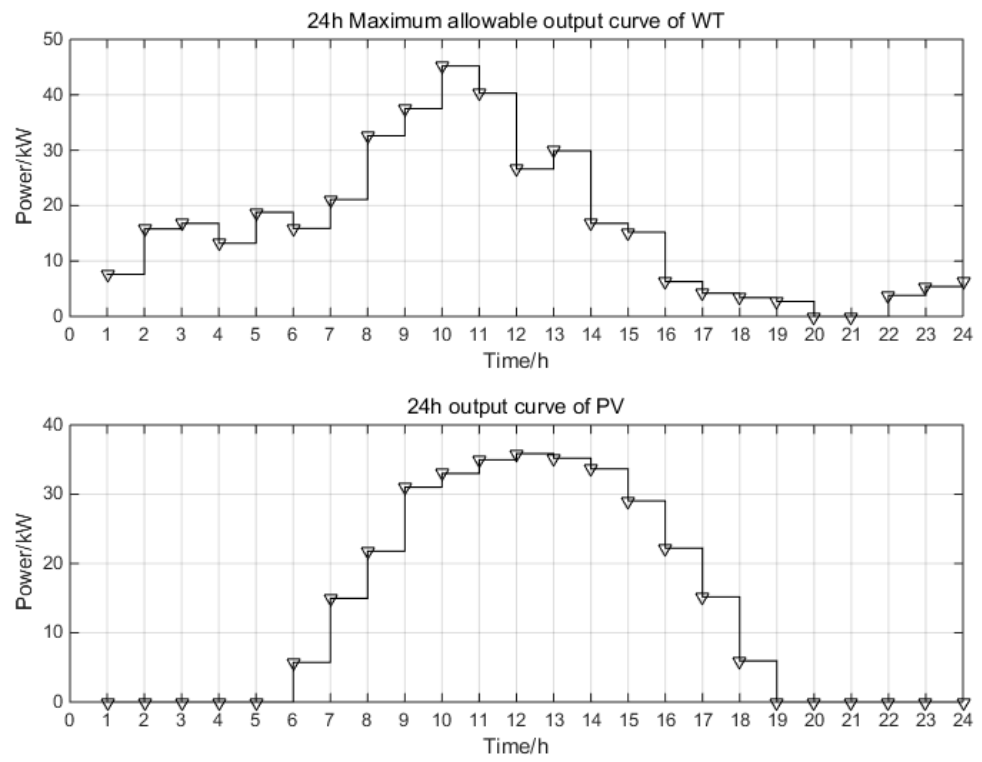

Figure 2. Predicted the output of $W T$ and $P V$ in a day. 


\subsection{Simulation Results and Discussion}

In order to verify the optimization ability of the IBBO algorithm, IBBO and basic BBO, as well as classic optimization algorithms PSO and GA [26], simulated the typical standard function [27] and compared the test results. The dimensions of each test function are all 30 dimensions, the maximum number of iterations is 1000, and the predetermined error of the function solution is $1.0 \times 10^{-8}$. the representative and difficult-to-solve Ackley and Griewank functions can be taken as examples. The average value, standard deviation and the number of function evaluations and success rates obtained when the solution accuracy is reached are shown in Table 2. Among them, the success of the problem solving is defined as the algorithm's convergence accuracy before the maximum function evaluation times reach a predetermined error, each algorithm runs independently 30 times and the maximum function evaluation times is $1.0 \times 10^{6}$.

Table 2. Algorithm performance comparison.

\begin{tabular}{cccccc}
\hline Function & Algorithm & Average Value & $\begin{array}{c}\text { Standard } \\
\text { Deviation }\end{array}$ & $\begin{array}{c}\text { Function } \\
\text { Evaluation Times }\end{array}$ & Success Rate \\
\hline \multirow{3}{*}{ Ackley } & IBBO & $1.1949 \times 10^{-12}$ & $4.7684 \times 10^{-23}$ & 140,640 & 100 \\
& PSO & $6.1748 \times 10^{-3}$ & $3.2709 \times 10^{-6}$ & 155,850 & 90 \\
& GA & $5.7820 \times 10^{-2}$ & $3.6079 \times 10^{-3}$ & 536,120 & 73.33 \\
Griewank & BBO & $7.1061 \times 10^{-1}$ & $2.6396 \times 10^{-1}$ & $1,329,270$ & 23.33 \\
& IBBO & $7.3121 \times 10^{-13}$ & $3.6763 \times 10^{-27}$ & 124,320 & 100 \\
& PSO & $1.2079 \times 10^{-4}$ & $3.8624 \times 10^{-6}$ & 105,810 & 100 \\
\hline
\end{tabular}

It can be seen from Table 2 that compared with the PSO, GA and basic BBO, the improved IBBO algorithm can achieve better convergence accuracy and stability. At the same time, the number of function evaluations used to achieve solution accuracy is also less. It shows that the IBBO algorithm has certain advantages in convergence accuracy, convergence speed and robustness compared with other algorithms, and has a good global search ability.

According to the dispatching strategy and the mathematical model built of the microgrid under the grid-connected mode, the operation state of the distributed generation for the typical summer microgrid is analyzed and solved using the IBBO algorithm. After optimized scheduling, the corresponding optimized output curve with non-load shifting of each micro power at each time is shown in Figure 3, and the comparison of the maximum allowable output and actual output power of WT is given in Figure 4. 


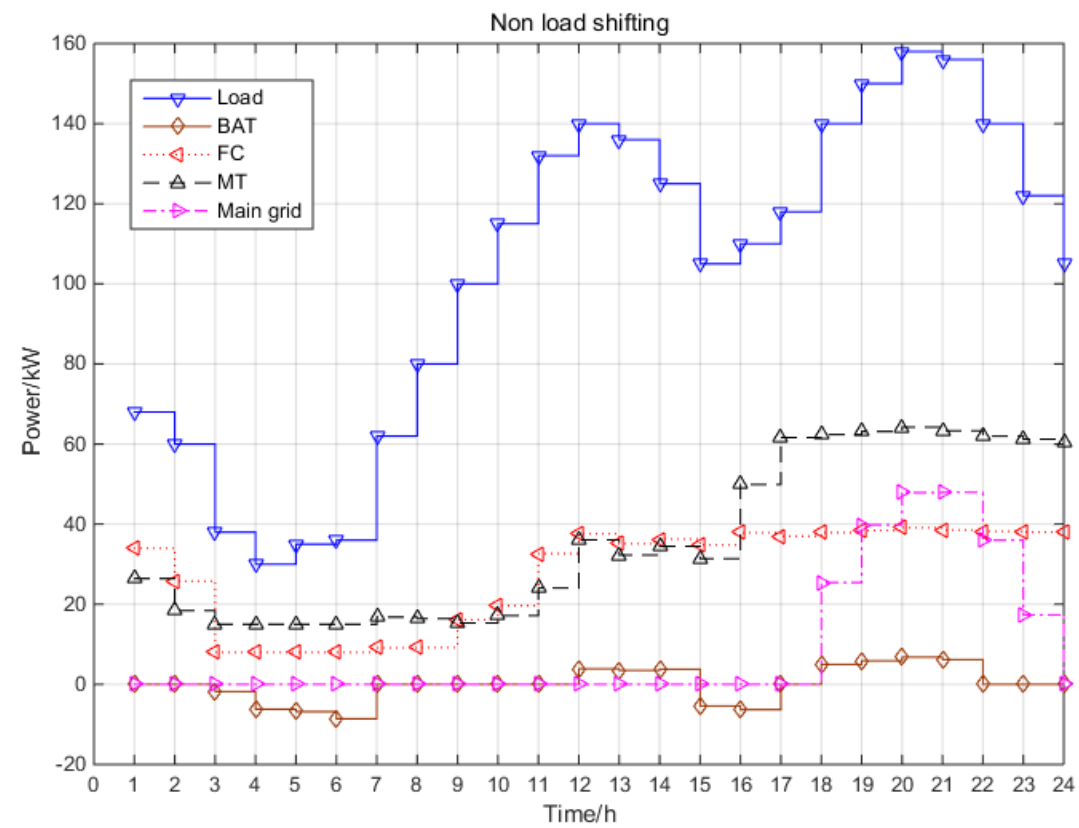

Figure 3. The comprehensive optimal scheduling results with non-load shifting.

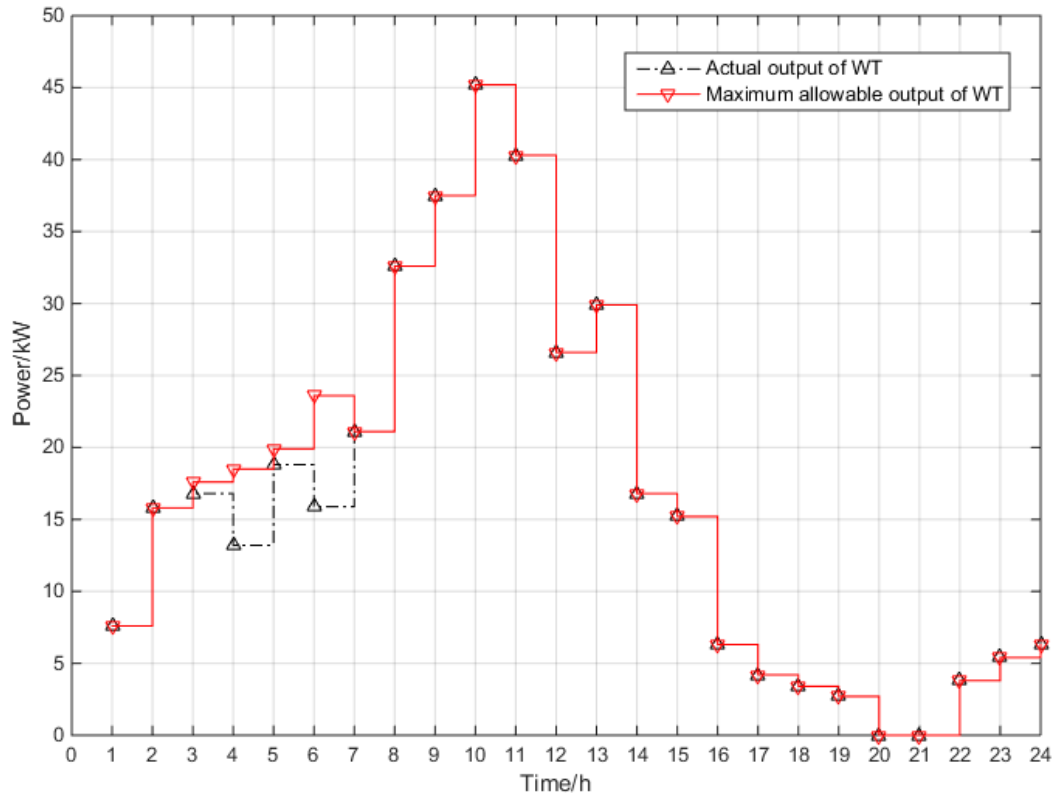

Figure 4. Comparison of the maximum output and actual output of WT.

In Figure 3, it is necessary to divide a day into several periods for analysis. The electricity valley time in the whole day is from 0:00 to 6:00, and its power demand can be as low as $30 \mathrm{~kW}$; the electricity purchase cost to the main grid is relatively low. There is only weak light at 6:00 and $P V$ provides hardly any output power, but there is wind power, and WT can continuously provide more than $20 \mathrm{~kW}$ of electric power. Due to the reasonable allocation of power resources, FC and MT operate at the lowest power, and the battery remains charged during the low power consumption period. Then, priority is given to purchasing electricity from the main grid to meet the load demand. Additionally, in combination with Figure 4, it can be seen that from 3:00 to 6:00, although the load power demand is met, the battery output power has been negative; therefore, the battery has been charging and due to its low power consumption and the capacity limitation of the battery, 
the actual output power of the WT is less than its maximum allowable output, resulting in a waste. After 7:00, the power consumption begins to increase gradually, and the light intensity gradually increases. From 7:00 to 10:00, the output of $P V$ and WT combined with the low-power operation of $F C$ and $M T$ can basically meet the load power demand. From 10:00 to 14:00, with the gradual increase in power consumption, the output of $W T$ and $P V$ no longer meet the current load demand. Meanwhile, FC is almost close to full capacity, and $M T$ also maintain the higher power operation. The battery is discharged during this period from 12:00 to 14:00, the peak time of electricity consumption. The local electricity valley time is from 15:00 to 16:00, which reaches the electricity valley bottom at 15:00. After the morning peak discharge, the battery is recharged during this period to store energy for the peak power consumption in the evening. After 17:00, the load demand begins to rise again, and at 20:00, the load reaches the peak of the whole day. Meanwhile, the light intensity gradually weakens, $P V$ basically no longer has an output. From the wind speed forecast for the day, the wind speed is also currently low, although the power supply of $W T$ is sustainable but very small. In this period, $F C$ and $M T$ operate at maximum power. Furthermore, the battery also discharges to meet the peak of power consumption. However, this period is the peak of the whole day, the total generation capacity of the microgrid is lower than the load demand; therefore, it is still necessary to purchase electricity from the main grid. After 23:00, the electricity consumption decreases, the $F C$ and $M T$ operate at reduced power, and the remaining capacity of the battery has also reached its lower limit.

Under the condition of $P V$ and WT output all day, FC and MT are used to power generation with power purchase from the main grid to meet the all-day load demands. $F C, \mathrm{O}$ and $\mathrm{M}$ factors and environmental costs are significantly lower than MT due to a combination of economic and environmental costs, although $F C$ also has higher fuel costs, but the comprehensive costs are significantly lower than $M T$. Therefore, in addition to the price of electricity valley time, $F C$ is close to full load power generation, playing a major role. Meanwhile, the battery is charging in the low valley, while discharging in the peak, which reflects the value of cutting peaks and filling valleys and stabilizing fluctuation.

According to the proposed optimization scheduling model, the daily comprehensive operating cost calculated by IBBO is compared with $\mathrm{BBO}$ and the other two algorithms, and four algorithms are independently run 30 times to obtain the average value. The comparison results are shown in Figure 5.

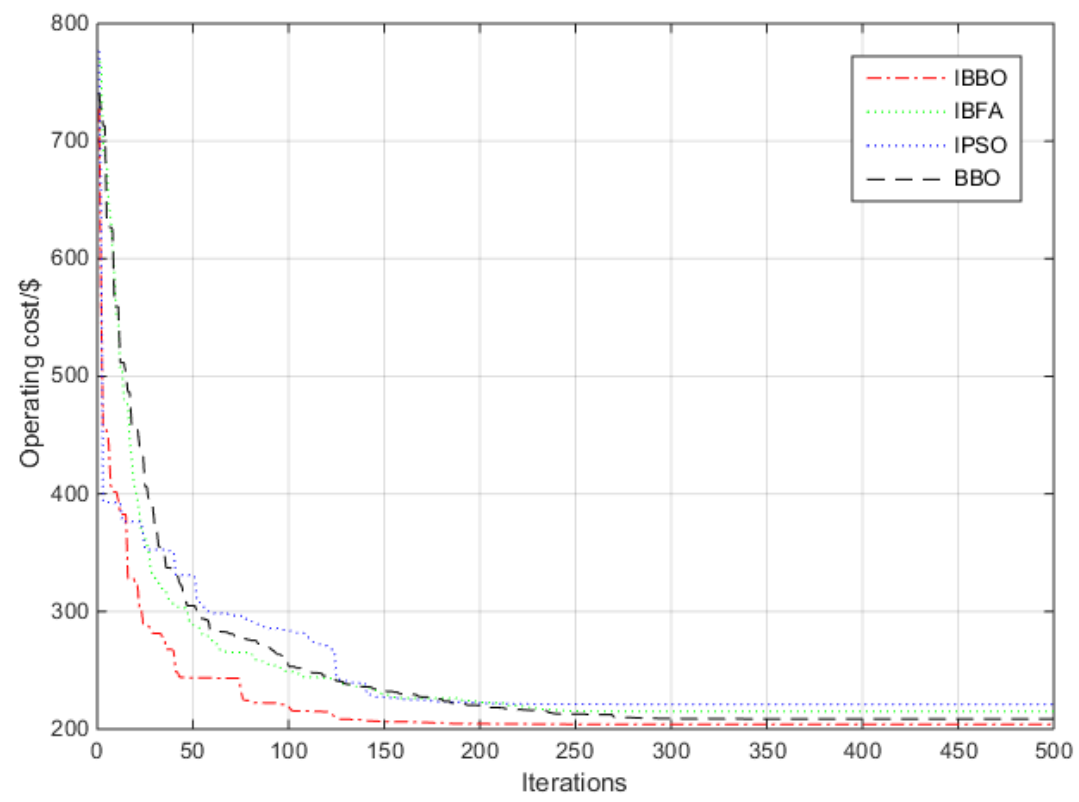

Figure 5. Comprehensive cost by 4 algorithms. 
It can be known from Figure 5, compared with IBFA, IPSO and BBO algorithms, that the IBBO algorithm has already converged in the 161st generation, and the convergence value of USD 204 is better than the other three algorithms; therefore, it can be concluded that the IBBO algorithm has great advantages in optimization accuracy, convergence speed and stability under the condition of the same population size and iteration times.

At the same time, the performance of the four algorithms in the calculation of the operating cost of the microgrid is evaluated. The comparison results are given in Table 3.

Table 3. Comparison of optimization algorithms for calculating operating costs.

\begin{tabular}{cccc}
\hline Optimization Method & $\begin{array}{c}\text { Maximum Iteration } \\
\text { Value/Times }\end{array}$ & $\begin{array}{c}\text { Convergence } \\
\text { Iterations/Times }\end{array}$ & $\begin{array}{c}\text { Operating } \\
\text { Costs/USD }\end{array}$ \\
\hline IBFA & 500 & 263 & 223 \\
IPSO & 500 & 212 & 218 \\
BBO & 500 & 275 & 212 \\
IBBO & 500 & 161 & 204 \\
\hline
\end{tabular}

Obviously, in Table 3, the number of convergence iterations of the optimization algorithm proposed in this paper is significantly less than the other three algorithms. This is mainly due to the fact that the IBBO algorithm normalizes the individuals of each generation on the basis of the $\mathrm{BBO}$ algorithm and adds disturbance processing to the migration mechanism to speed up the optimization speed and reduce convergence iterations of the algorithm, which also makes it easier to obtain the optimal value of the operating cost from the IBBO algorithm.

Next, we consider the case where the interruptible load can perform power shifting in a microgrid operation. Suppose that the interruptible load can be power shifted, compared with the maximum power demand in the original period, the variation of the hourly power demand shifted $P_{L S m}$ is not more than $20 \mathrm{~kW}$, and the total amount of shiftable power for the interruptible load $P_{L I}$ is not more than $80 \mathrm{~kW}$ in the whole day. Meanwhile, the effect of the shifting time quanta on the scheduling results does not need to be considered. The load shifting curve and the WT output curve without considering shifting time are given in Figure 6, and the optimization results of each micro-energy source is given in Figure 7.
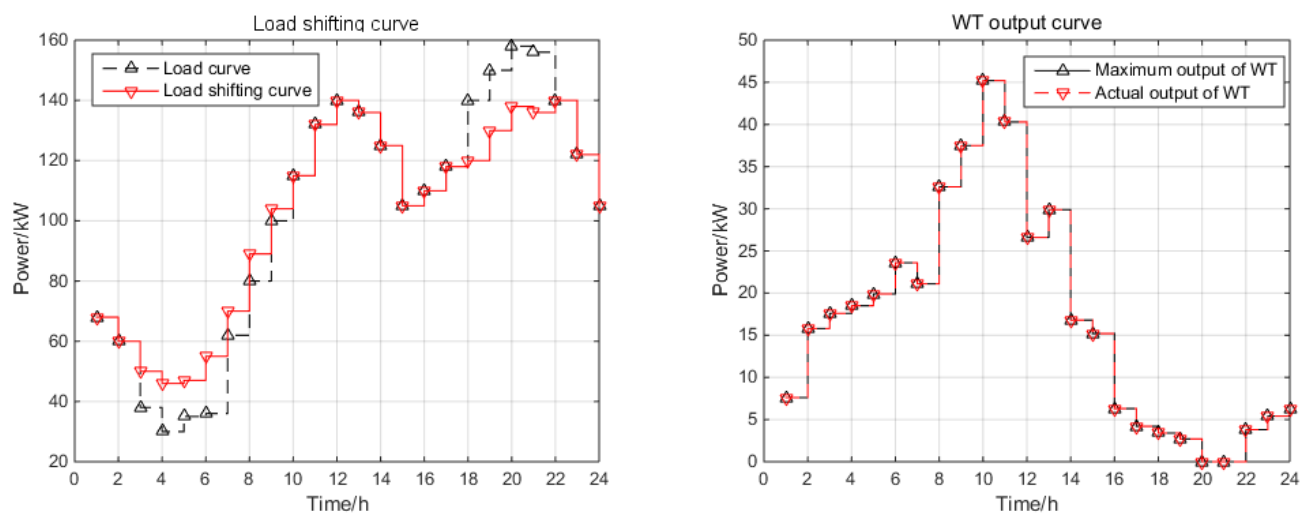

Figure 6. Output curves of the load shifting and WT while taking no account of shifting time. 


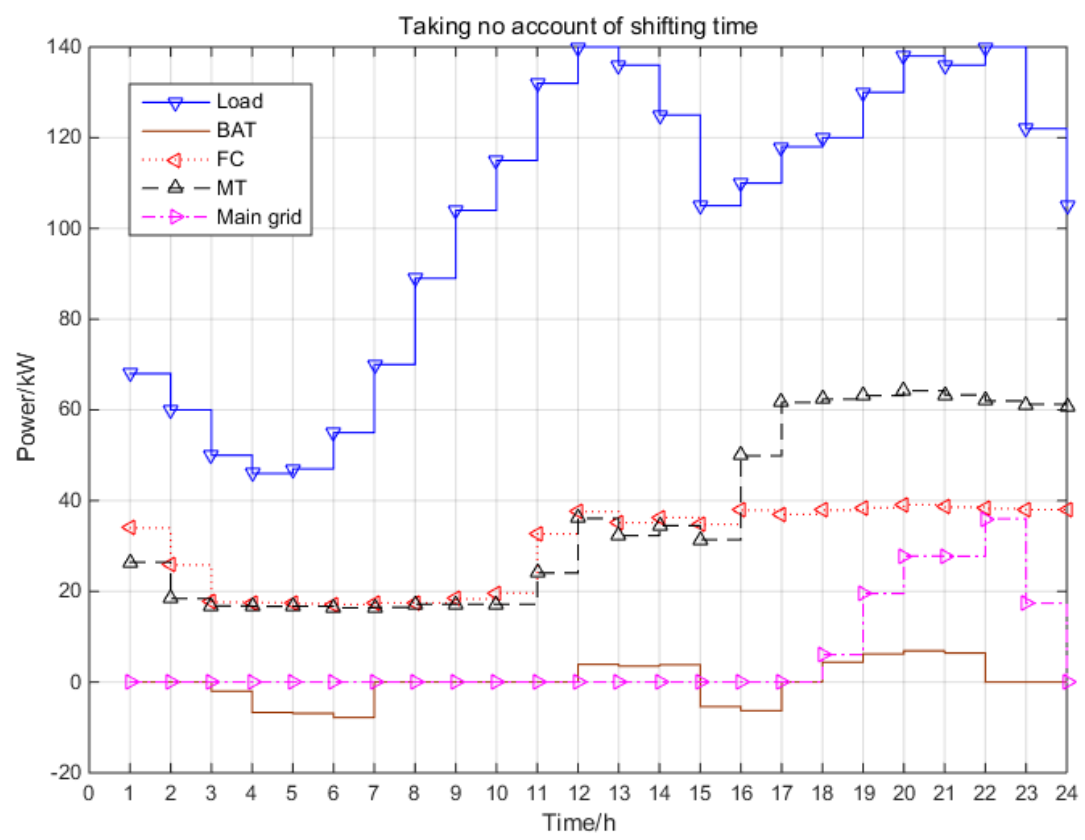

Figure 7. Results of the comprehensive optimal scheduling while taking no account of shifting time.

From the load shifting curve in Figure 6, the peak load power of the whole day before the load shift reaches the maximum value of $160 \mathrm{~kW}$ from 18:00 to 21:00, which is also the period with low $P V$ and $W T$ power output and the period with the greatest amount of electricity purchased on that day. In addition, the load power valley drops to the minimum value of $30 \mathrm{~kW}$ from 3:00 to 7:00. The power of interruptible load can be shifted to the low power consumption period of the whole day from 3:00 to 9:00, and the shift amount is the greatest from 3:00 to 6:00. After the load is shifted, the load power is cutting the peak and filling the valley, reducing the burden of power generation on the microgrid. As can be seen from the WT output curve in Figure 6 after power shifting, the maximum allowable output of WT is exactly in line with the actual output power, and there is no longer any wastage.

It can be seen from Figure 7 that $F C$ and $M T$ can operate at low power from 0:00 to 11:00, which can meet the load power demand with the power generation of $P V$ and WT and recharge the batteries in the low power consumption period. After 12:00, the power generation of $F C$ and $M T$ increases gradually, and the power is close to full output during the peak period in the evening. Although there is still a need to purchase power from the main grid from 19:00 to 23:00 due to the lack of power generation in the microgrid, the power purchase has been significantly reduced compared to the operation of the uninterruptible load, i.e., Figure 3.

Then, we consider the case that power shifting can be performed, and the shifting time needs to be considered. With the other conditions remaining unchanged, suppose that the effect of the shifting time quanta on optimal scheduling needs to be considered, the load shifting curve and the WT output curve are given by Figures 8 and 9, and the corresponding optimal output of each micro power supply is indicated. 

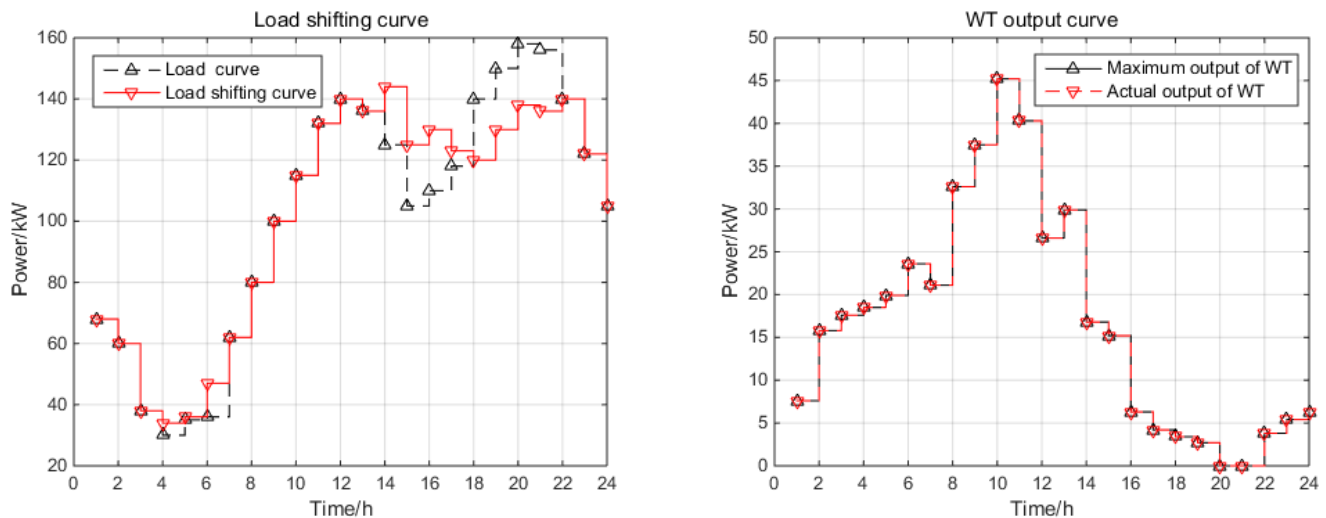

Figure 8. Output curves of the load shifting and WT while taking account of shifting time.

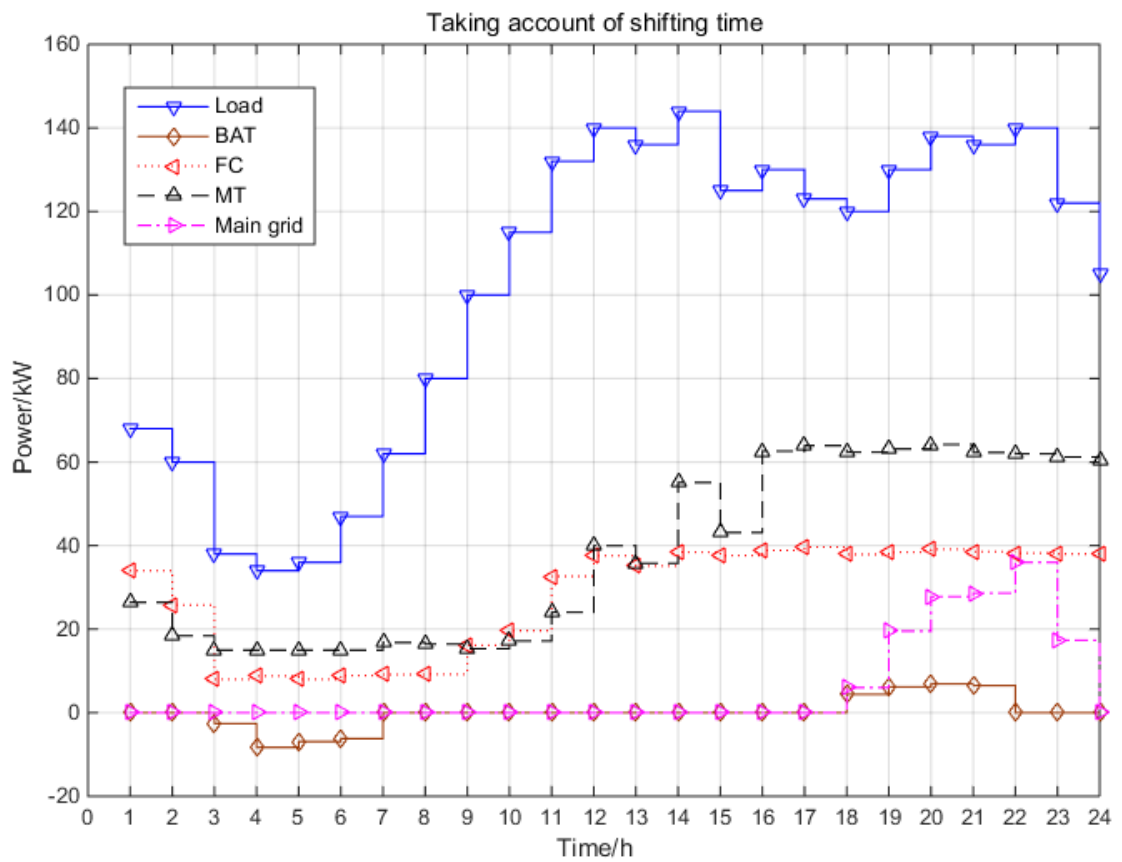

Figure 9. Results of the comprehensive optimal scheduling while taking account of shifting time.

Since the effect of the shifting time of interruptible load on the comprehensive evaluation is considered, the comparison of the load shifting curves between Figures 6 and 8 shows that the shiftable power from 18:00 to 21:00 in Figure 6 is mainly shifted toward the morning period of the low power consumption of the whole day. Whereas only a fraction of the power is shifted to the period from 4:00 to 6:00 in Figure 8, most of the remaining power is shifted to the period from 14:00 to 17:00. There are two main reasons. Firstly, although the interruptible load is shifted from the evening peak period to the period between 4:00-6:00, which has a higher time cost in terms of shifting, the comprehensive cost is low because there is a situation where the WT power is abandoned during this period, resulting in the wasted part of this power being basically cost-free with only a time cost. Secondly, 13:00-17:00 is a local valley period in the whole day and shifting the evening peak power consumption to this period only increases the output power of $F C$ and $M T$, because $W T$ and $P V$ are always outputting power during this time period, the load power demand after the shifting of microgrid can be met and the time cost of power shifting is low.

As can be seen from the comparison between Figures 7 and 9, due to the shift of interruptible load in the evening peak, the period of local low power consumption, which 
originally occurred from 15:00 to 16:00, no longer exists. The battery is only charged during the valley period from 3:00 to 6:00, and it discharges in the evening peak period, in order to meet the shifting load demand, the output power of the fuel cell is increased, while the number of battery charge and discharge cycles is reduced, and the charge and discharge loss of the battery is reduced, thereby reducing the overall operating cost.

Comparing the above cases without considering load shifting and considering load shifting, the comprehensive operation cost including economic cost and environmental cost is calculated in Table 4.

Table 4. Cost of non-load shifting and load shifting.

\begin{tabular}{ccc}
\hline Scenario & Case & $\begin{array}{c}\text { Comprehensive Operating } \\
\text { Cost/USD }\end{array}$ \\
\hline 1 & Non-load shifting & 204.06 \\
2 & Taking no account of shifting time & 190.34 \\
3 & Taking account of shifting time & 195.46 \\
\hline
\end{tabular}

In Table 4, the optimal comprehensive operating costs without considering interruptible load and considering interruptible load are given, respectively. Without considering interruptible load, the comprehensive operating cost is 204 US dollars, which is the highest among the three scheduling scenarios. When interruptible load is considered, the power generation of $P V$ and $W T$ is no longer wasted due to power shifting, and the coordinated generation of $F C$ and $M T$ has also changed; therefore, the comprehensive operating cost can be relatively reduced. Comparing scenarios two and three, in scenario two, the interruptible load shifts to the place where the power of the whole day is lower due to not considering the effect of the shifting time, and the scheduling scheme of the optimal comprehensive cost by meeting the conditions is given. In contrast, in scenario three, the inclusion of the shifting time causes the interruptible load to shift to the time with lower power, which is closer to the original operation time, and the comprehensive operating cost of scheme three is slightly higher than that in scenario two, but lower than that in scenario one.

Consequently, in microgrid operation, for the presence of the power shifting of interruptible load, considering the effect of environmental benefits and economic costs, and to ensure that the capacity constraints of the battery are met, we can conclude that the comprehensive operating cost after shifting interruptible load can be lower, and the time factor $\alpha$ in this paper is proposed to adjust the relationship between the power shifting time and the user acceptability. From the comparative results, on the user side, adopting reasonable load management schemes and power demand incentive measures can effectively improve the utilization rate of renewable energy and ensure the economy and effectiveness of the microgrid system operation.

\section{Conclusions}

This paper proposes a multi-objective optimal dispatching model of distributed power generation in the microgrid grid-connected mode that combines operating costs and environmental benefits. Meanwhile, on the user side, the power shifting for interruptible load is joined considering the effect of interruptible load on the operating cost of a microgrid based on battery storage capacity. Based on the proposed IBBO algorithm, a typical microgrid, as an example, containing a fuel cell, micro turbine, wind, storage battery, light and load is used to optimize the output of each distributed generator to achieve the maximum economic and environmental benefits, and the results obtained by comparing and analyzing another three optimization algorithms prove the superiority of the IBBO algorithm. Moreover, the optimization performance of $\mathrm{BBO}$ is improved by better designing the migration strategy and the determination principle of migration rate, and the reliability and applicability of the proposed model and algorithm are verified. This optimization operation method based on an improved biogeographical optimization algorithm can 
improve the level of microgrid operation technology. If we can properly consider the power shifting of interruptible load, effectively formulate the incentive measures for the user side, improve the management of the demand side and guide the load effectively, the maximum utilization of renewable energy can be achieved effectively, which is of certain significance to the maximization of the operation benefits of the microgrid.

Author Contributions: Data curation, B.L.; software, J.W.; validation, H.D.; methodology, B.L.; writing-original draft, B.L.; writing-review and editing, H.D. and J.W.; visualization, H.D.; supervision, J.W. All authors have read and agreed to the published version of the manuscript.

Funding: This research received no external funding.

Conflicts of Interest: The authors declare no conflict of interest.

\section{References}

1. Chen, J.J.; Qi, B.X.; Rong, Z.K.; Peng, K.; Zhao, Y.L.; Zhang, X.H. Multi-energy coordinated microgrid scheduling with integrated demand response for flexibility improvement. Energy 2020, 217, 119387. [CrossRef]

2. Kiptooa, M.K.; Lotfy, M.E.; Adewuyi, O.B.; Conteh, A.; Howlader, A.M.; Senjyu, T. Integrated approach for optimal technoeconomic planning for high renewable energy-based isolated microgrid considering cost of energy storage and demand response strategies. Energy Convers. Manag. 2020, 215, 112917. [CrossRef]

3. Yong, L.; Zou, Y.; Tan, Y.; Cao, Y.; Liu, X.; Shahidepour, M.; Tian, S.; Bu, F. Optimal Stochastic Operation of Integrated Low-Carbon Electric Power, Natural Gas, and Heat Delivery System. IEEE Trans. Sustain. Energy 2018, 9, 273-283.

4. Samy, M.M.; Barakata, S.; Ramadan, H.S. Techno-economic analysis for rustic electrification in Egypt using multi-source renewable energy based on PV/wind/FC. Int. J. Hydrogen Energy 2020, 45, 11471-11483. [CrossRef]

5. Tanmay, D.; Ranjit, R.; Krishna, M.K. Impact of the penetration of distributed generation on optimal reactive power dispatch. Prot. Control Mod. Power Syst. 2020, 5, 2-26.

6. Takano, H.; Goto, R.; Hayashi, R.; Asano, H. Optimization Method for Operation Schedule of Microgrids Considering Uncertainty in Available Data. Energies 2021, 14, 2487. [CrossRef]

7. Lingmin, C.; Jiekang, W.; Fan, W.; Huiling, T.; Chanjie, L.; Yan, X. Energy flow optimization method for multi-energy system oriented tocombined cooling, heating and power. Energy 2020, 211, 118536. [CrossRef]

8. Tantrapon, K.; Jirapong, P.; Thararak, P. Mitigating microgrid voltage fluctuation using battery energy storage system with improved particle swarm optimization. Energy Rep. 2020, 6, 724-730. [CrossRef]

9. Nemati, M.; Braun, M.; Tenbohlen, S. Optimization of unit commitment and economic dispatch in microgrids based on genetic algorithm and mixed integer linear programming. Appl. Energy 2018, 210, 944-963. [CrossRef]

10. Nagapurkar, P.; Smith, J.D. Techno-economic optimization and environmental Life Cycle Assessment (LCA) of microgrids located in the US using genetic algorithm. Energy Convers. Manag. 2019, 181, 272-291. [CrossRef]

11. Roy, K.; Mandal, K.K.; Mandal, A.C.; Patra, S.N. Analysis of energy management in micro grid-A hybrid BFOA and ANN approach. Renew. Sustain. Energy Rev. 2018, 82, 4296-4308. [CrossRef]

12. Cunbin, L.; Jianye, Z.; Peng, L. Multi-objective Optimization Model of Micro-grid Operation Considering Cost, Pollution Discharge and Risk. Proc. CSEE 2014, 34, 3073-3079.

13. Chen, J.; Yang, X.; Zhu, L.; Zhang, M.; Li, Z. Microgrid multi-objective economic dispatch optimization. Proc. CSEE 2013, 33, 57-66. (In Chinese)

14. Chen, D.; Gong, Q.; Zhang, M.; Liu, D.; Du, L.; Shao, Q. Multi-objective optimal dispatch in wind power integrated system incorporating energy-environmental efficiency. Proc. CSEE 2011, 31, 10-17. (In Chinese)

15. Zhao, L.; Ju, G.; Lü, J. An improved genetic algorithm in multi-objective optimization and its application. Proc. CSEE 2008, 28, 96-102. (In Chinese)

16. Faisal, A.; Mohamed, H.; Koivo, N. Online management of microgrid with battery storage using multi-objective optimization. In Proceedings of the International Conference on Power Engineering, Energy and Electrical Drives, Setubal, Portugal, 12-14 April 2007; pp. 231-236.

17. Ma, X.; Wu, Y.; Fang, H.; Sun, Y. Optimal sizing of hybrid solar-wind distributed generation in an island micro grid using improved bacterial foraging algorithm. Proc. CSEE 2011, 31, 17-25.

18. Li, P.; Xu, W.; Zhou, Z.; Li, R. Optimal Operation of Microgrid Based on Improved Gravitational Search Algorithm. Proc. CSEE 2014, 34, 3073-3079.

19. Zheng, Z.; Ai, Q.; Xu, W.; Shi, J.; Xie, D.; Han, L. A multi-objective dispatch optimization strategy for economic operation of smart grids. Power Syst. Technol. 2010, 34, 7-13. (In Chinese)

20. Liu, D.; Guo, J.; Huang, Y.; Wang, W. Dynamic economic dispatch of wind integrated power system based on wind power probabilistic forecasting and operation risk constraints. Proc. CSEE 2013, 33, 9-15. (In Chinese)

21. Mohammadi, S.; Mozafari, B.; Solimani, S.; Niknam, T. An adaptive modified firefly optimization algorithm based on Hong's Point Estimate Method to optimal operation management in a microgrid with consideration of uncertainties. Energy 2013, 51, 339-348. [CrossRef] 
22. Simon, D. Biogeography-based optimization. IEEE Trans. Evol. Comput. 2008, 12, 702-713. [CrossRef]

23. Khurmi, R.S.; Sedha, R.S. Materials Science. S. Chand. 2008. Available online: https://refhub.elsevier.com/S0360-3199(18)31672 $-0 /$ sref36 (accessed on 2 September 2021).

24. Giovanni, C.; Luca, M. Resolution of Spike Overlapping by Biogeography-Based Optimization. Electronics 2021, 10, 1469.

25. Khosravi, K.; Bordbar, M.; Paryani, S.; Saco, P.M.; Kazakis, N. New hybrid-based approach for improving the accuracy of coastal aquifer vulnerability assessment maps. Sci. Total Environ. 2021, 767, 145416. [CrossRef]

26. Holland, J.H. Adaptation in Natural and Artificial Systems: An Introductory Analysis with Applications to Biology, Control, and Artificial Intelligence, 2nd ed.; MIT Press: Cambridge, MA, USA, 1992.

27. Rahnamayan, S.; Tizhoosh, H.R.; Salama, M.M.A. Opposition-based differential evolution. IEEE Trans. Evolut. Comput. 2008, 12, 64-79. [CrossRef] 